\title{
Surface-state hole decay mechanisms: The Be(0001) surface
}

\author{
V. M. Silkin, ${ }^{1}$ T. Balasubramanian, ${ }^{2}$ E. V. Chulkov, ${ }^{1,3}$ A. Rubio,,${ }^{1,3}$ and P. M. Echenique ${ }^{1,3}$ \\ ${ }^{1}$ Donostia International Physics Center (DIPC) and Centro Mixto CSIC-UPV/EHU, 20018 San Sebastián, Spain \\ ${ }^{2}$ MAX-Lab, Lund University, Lund S 22100, Sweden \\ ${ }^{3}$ Departamento de Física de Materiales, Facultad de Ciencias Químicas, Universidad del Pais Vasco/Euskal Herriko Unibertsitatea, \\ Apdo. 1072, 20018 San Sebastián/Donostia, Basque Country, Spain
}

(Received 16 April 2001; published 8 August 2001)

\begin{abstract}
First-principle calculations and angle-resolved photoemission experiments are combined to analyze the different contributions to the surface-state hole dynamics at the $\mathrm{Be}(0001)$ surface. The computed inelastic electron-electron scattering rate $\gamma$ is in good agreement with experiment once the electron-phonon contribution is subtracted. $\gamma$ is strongly influenced by details of the surface band structure. In particular, the intraband transitions within the surface state itself ignored in traditional Fermi liquid theory contribute $65 \%-85 \%$ to $\gamma$. This intraband contribution and the highly spatial nonlocality of the self-energy makes the energy and momentum dependence of $\gamma$ to be $\propto\left(E_{F}-E\right)^{\alpha}$ (with $\alpha<2$ ) in contrast to the observed $\alpha \simeq 2$ value in noble and transition metal surfaces.
\end{abstract}

PACS number(s): 73.20.At, 79.60.Bm, 79.60.Dp, 79.60.Ht

Many-body electron interactions are ubiquitious in all physical processes of fundamental and technological interest in surface science. In particular, the interplay between electron-electron interaction, electron-phonon interaction, and surface morphology is of key importance to explain the complex electron dynamics and quantum interference at surfaces including charge and energy transfer. Among other effects these interactions introduce a finite lifetime, $\tau$, that characterizes how long the quantum state corresponding to a quasiparticle, an elementary excitation of the interacting Fermi liquid, retains its identity.

Surface states are very sensitive to local changes at the surface and are ideal to discriminate between screening, localization and vibrational contributions to the quasiparticle dynamics. These two-dimensional (2D) states, observed on metal surfaces, ${ }^{1}$ have an increasing importance in both surface science ${ }^{2}$ and nanoscale technology. ${ }^{3,4}$ In spite of this inherent relevance our fundamental understanding of the mechanisms controlling the electron/hole surface dynamics is still lacking. For instance, theoretical models based on a homogeneous 3D (2D) Fermi liquid ${ }^{5,6}$ predict a substantially longer (shorter) lifetime for surface-state holes than that observed with both photoemission spectroscopy (PES) (Refs. 7 and 8) and scanning tunneling spectroscopy (STS). ${ }^{9,10}$ By resorting to the different dimensionality of surface states and bulk screening, the authors in Ref. 10 reconcile this controversy within a one-dimensional model calculation of the hole decay only at the zone center $(\bar{\Gamma})$. However the physically relevant energy and momentum dependence of $\tau$ is missing. Recent PES and STS measurements of the energy dependence of the lifetime for the occupied $d$-surface state on Mo(110) (Ref. 8) and unoccupied $s p$-surface state on $\mathrm{Cu}(111)$ and $\mathrm{Ag}(111)$ (Ref. 11) follow a quadratic energy dependence, considered as a fingerprint of Fermi liquid behavior. This is widely-used in angle-resolved photoemission studies to extract information about electron correlations and phase transitions in different compounds, including superconductors. ${ }^{4}$ From those results one is tempted to assume that quadratic dependence is a common law for all metals and that any deviation is a signature of electron cor- relation or more complex phenomena. In the present letter we show that this is not generally true by performing detailed ab initio calculations of the hole decay in different surface states on $\mathrm{Be}(0001)$. The highly spatial nonlocality of the electron self-energy and the peculiar surface band structure provides the basic framework to understand hole decaying surface processes at metal surfaces.

The surface states on $\mathrm{Be}(0001)$ has been studied extensively both experimentally ${ }^{12}$ and theoretically. ${ }^{13}$ These states form a high density 2D electron gas with a high surface density of states (DOS) at the Fermi level $E_{F}$ (Ref. 13) that explains many unusual properties of $\mathrm{Be}(0001),{ }^{14}$ among them the anomalously large electron-phonon coupling at the surface. ${ }^{15}$ Figure 1 shows the surface electronic structure of $\mathrm{Be}(0001)$ from a self-consistent local density-functional plane-wave pseudopotential calculation using a supercell of 18 atomic layers of $\mathrm{Be}(0001)$ and 6 layers of vacuum. This supercell is big enough to ensure a good description of both surface and bulk states. ${ }^{16}$ The computed surface band structure is in excellent agreement with (PES) data. ${ }^{12,15}$ So renormalization of the quasiparticle energies should be small and, therefore, the $\mathrm{Be}(0001)$ surface is an ideal system to directly investigate the decay mechanisms of $2 \mathrm{D}$ quasiparticles at metal surfaces.

The evaluation of the electron-electron scattering contribution to the linewidth $\gamma=\tau^{-1}$ was performed within the first-principles GW self-energy formalism. ${ }^{17}$ This technique has been successful in describing electron/hole lifetimes in noble and simple bulk metals. ${ }^{18}$ Here we extend the formalism to deal with nonperiodic system such as surfaces and include the specific surface response in evaluating the dynamical screening. From the computed ground-state electronic structure we evaluate the damping rate of an initial state $\psi_{0}$ through (we use atomic units, i.e., $e^{2}=\hbar=m_{e}=1$ )

$$
\begin{aligned}
\gamma= & -\frac{2}{\Omega} \sum_{\substack{n, \mathbf{k}_{\|} \\
E_{0} \leqslant E_{n \mathbf{k}_{\|}} \leqslant E_{F}}} \sum_{\mathbf{g}, \mathbf{g}^{\prime}, \mathbf{g}^{\prime \prime}, \mathbf{g}^{\prime \prime \prime}} c_{0}^{*}(\mathbf{g}) c_{n}^{*} \\
& \times\left(\mathbf{k}_{\|}+\mathbf{g}^{\prime}\right) \operatorname{Im} W_{\mathbf{g}^{\prime \prime}-\mathbf{g}, \mathbf{g}^{\prime \prime \prime}-\mathbf{g}^{\prime}}\left(\mathbf{k}_{\|} ; E_{n} \mathbf{k}_{\|}\right) c_{n}\left(\mathbf{k}_{\|}+\mathbf{g}^{\prime \prime}\right) c_{0}\left(\mathbf{g}^{\prime \prime \prime}\right),
\end{aligned}
$$




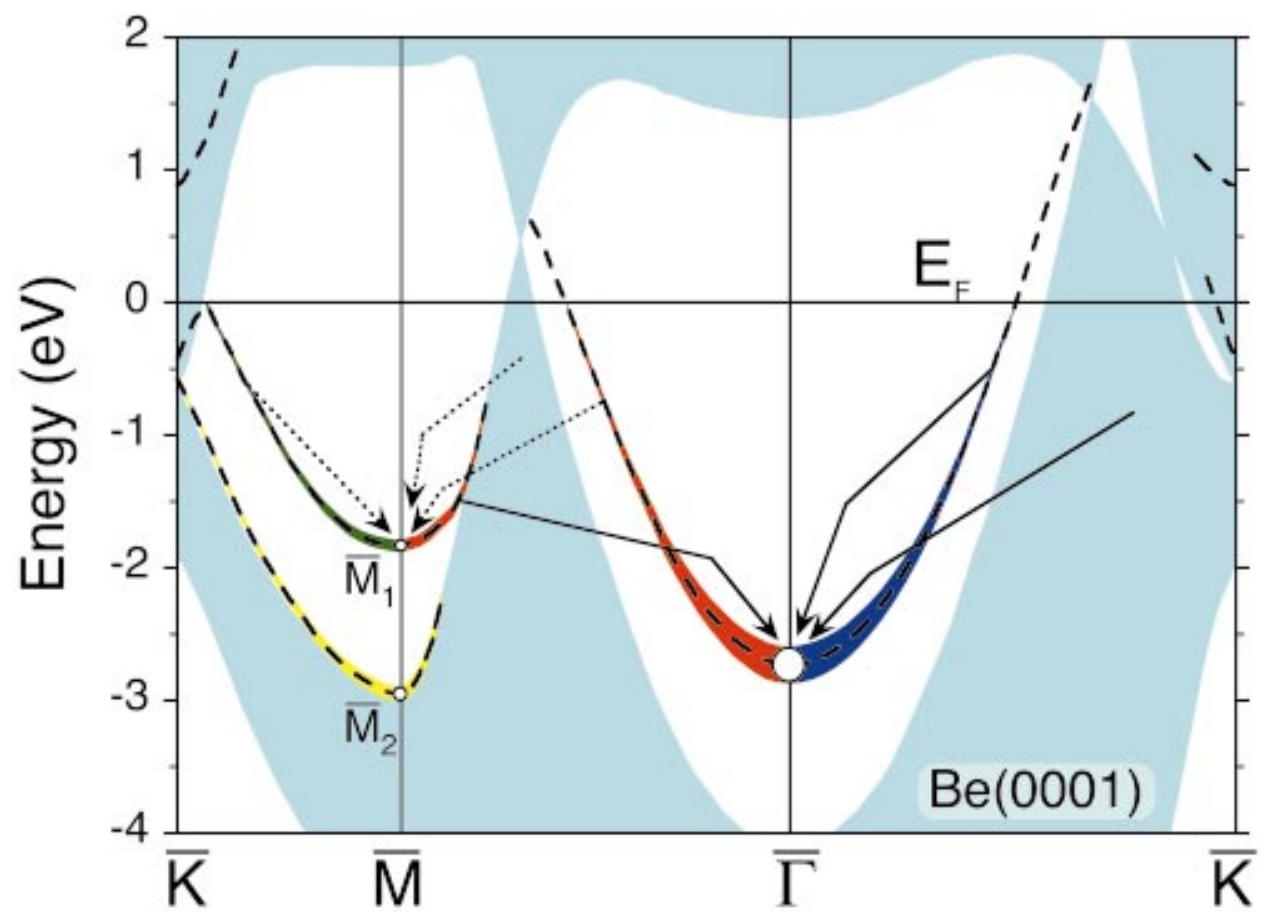

FIG. 1. (Color) The computed $\mathrm{Be}(0001)$ electronic structure along symmetry directions. The projected bulk states are in blue whereas the dashed lines denote the surface states. All possible electronic transitions to a surface hole (open circle) are indicated by arrows. The calculated linewidths are shown by colored bands as a function of the momentum. where $\mathbf{k}_{\|}$belongs to the first 2D Brillouin zone, $c_{n}\left(\mathbf{k}_{\|}+\mathbf{g}\right)$ are the Fourier coefficients of the initial and final wavefunction, and $\mathbf{g}$ are the reciprocal lattice vectors. The screened interaction $W_{\mathbf{g}, \mathbf{g}^{\prime}}\left(\mathbf{k}_{\|}, \omega_{n \mathbf{k}_{\|}}\right)$is computed within the random phase approximation. ${ }^{17}$ Computing $\gamma$ for pure bulk states ${ }^{18}$ we determined the convergence of our supercell calculation to be less than $5 \mathrm{meV}$.

The results of the present letter namely, the different contributions to the surface-state hole linewidth at $\bar{\Gamma}$ and $\bar{M}$ and its energy (momentum) dependence are summarized in Table I and Fig. 2. As follows from Table I, the computed $\gamma$ at $\bar{\Gamma}$ is in good agreement with the experimental value once the electron-phonon contribution is subtracted (see below) and with the $1 \mathrm{D}$ calculation linewidth. ${ }^{10}$ For the $\bar{M}_{1}$ state we found $\gamma=72 \mathrm{meV}$ that is significantly smaller than the old measured room temperature value of $380 \mathrm{meV}$ (Ref. 12) which contains large contributions from phonons, defects, and uncertainties due to experimental energy and momentum

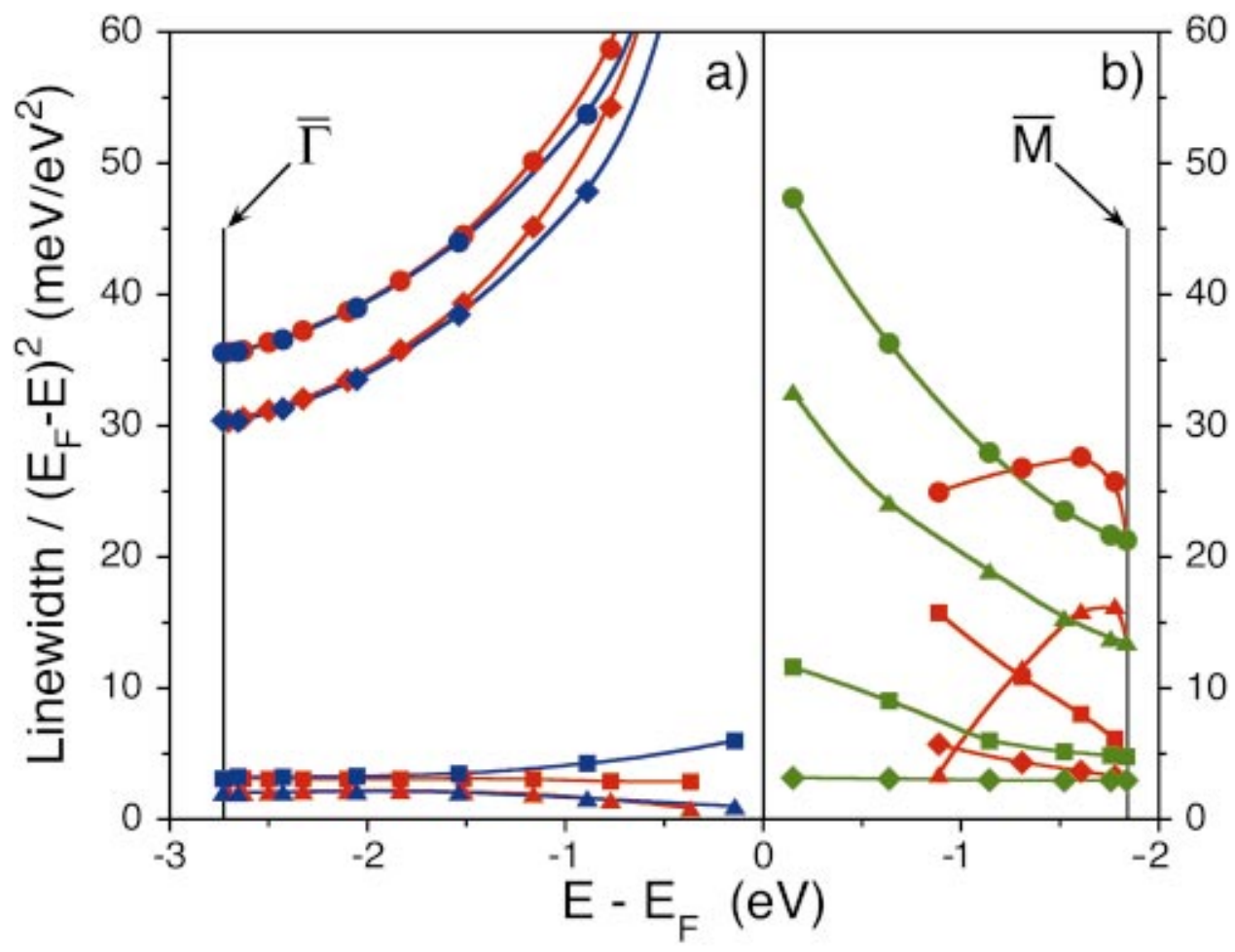

FIG. 2. (Color) The hole linewidth divided by $\left(E_{F}-E\right)^{2}$ as a function of energy for (a) the $\bar{\Gamma}$ surface state along $\overline{\Gamma M}$ (red) and $\overline{\Gamma K}$ (blue); (b) the $\bar{M}_{1}$ surface state along $\overline{M \Gamma}$ (red) and $\overline{M K}$ (green). Filled circles correspond to the total linewidth, diamonds give the contribution from the $\bar{\Gamma}$ state, squares from bulk states, and triangles from the $\bar{M}_{1}$ state. 
TABLE I. Surface-state linewidth $\gamma(\mathrm{meV})$ calculated at the $\bar{\Gamma}$ and $\bar{M}$ symmetry points together with the results from the 3D- and 2D-DEGM and experiments. We have decomposed $\gamma$ in contributions from different states. The computed surface state energies are in good agreement with experiments (in parenthesis). The lower line shows the 1D calculation results (Ref. 10).

\begin{tabular}{|c|c|c|c|c|c|c|c|c|c|}
\hline $\begin{array}{l}\text { Surface } \\
\text { state }\end{array}$ & $\begin{array}{l}\text { Surface state } \\
\text { energies }(e V)\end{array}$ & $\begin{array}{l}\text { from the } \\
\bar{\Gamma} \text { state }\end{array}$ & $\begin{array}{l}\text { Contribution } \\
\text { from the } \\
\bar{M}_{1} \text { state }\end{array}$ & $\begin{array}{l}\gamma \text { to } \gamma \text { (me) } \\
\text { from the } \\
\bar{M}_{2} \text { state }\end{array}$ & $\begin{array}{l}\text { from bulk } \\
\text { states }\end{array}$ & $\begin{array}{l}\text { Total decay } \\
\text { rate } \gamma \\
\text { (linewidth) }\end{array}$ & 3D-DEGM & 2D-DEGM & $\begin{array}{c}\text { Experiment } \\
(T=0 K)\end{array}$ \\
\hline $\bar{\Gamma}$ & $-2.73(-2.78)$ & 225 & 5 & $\sim 0$ & 35 & 265 & 90 & 610 & 281 \\
\hline $\bar{M}_{1}$ & $-1.83\left(-1.80^{12}\right)$ & 10 & 46 & $\sim 0$ & 16 & 72 & 40 & 420 & \\
\hline $\bar{M}_{2}$ & $-2.95\left(-3.0^{12}\right)$ & 9 & 36 & 9 & 42 & 96 & 105 & 620 & \\
\hline $\bar{\Gamma}$ & -2.80 & 240 & & & 40 & 280 & & & \\
\hline
\end{tabular}

resolution. Surprisingly, the calculated $\gamma$ of the $\bar{M}_{2}$ state is only slightly larger than the one at $\bar{M}_{1}$ which is $1 \mathrm{eV}$ closer to $E_{F}$ and nearly three times smaller than the $\gamma$ of the $\bar{\Gamma}$ surface state which has a similar binding energy. To understand this unusual behavior we first clarify the role of surface and bulk states in the hole decay mechanism.

In Fig. 2 we analyze in detail the different contributions to $\gamma$. We divide the transitions appearing in Eq. (1) into intraband (i.e., within the surface state itself) and interband transitions (from bulk and other surface states) (see the sketch in Fig. 1). Intraband transitions turn out to be the main channel responsible for the hole decay accounting for $85 \%(65 \%)$ of the total $\gamma$ at $\bar{\Gamma}\left(\bar{M}_{1}\right)$ and with increasing relevance as the state approaches $E_{F}$ (see Fig. 2 for the evolution of $(\bar{\Gamma})$ and $\gamma\left(\bar{M}_{1}\right)$ along the symmetry directions). Special mention should be given to the evolution of the $\bar{M}_{1}$ state along $\overline{M \Gamma}$. Figure 1 shows how this state rapidly becomes a resonance in the projected bulk structure as it disperses towards $E_{F}$. This transition is also manifested in a change of the dominant channel for hole decay in $\gamma$ as we move away from the bottom of the surface band towards $E_{F}$, namely, from intraband (2D) to interband (3D bulk) with a non-negligible contribution from the $\bar{\Gamma}$ surface state. So $2 \mathrm{D}$ inter- and intraband transitions account for $\sim 80 \%$ of the full $\gamma$ at $\bar{M}_{1}$. This result demonstrates the competition between the $2 \mathrm{D}$ character of the surface states and the 3D screening and delocalization of the underlying bulk states in controlling the hole-decay dynamics at metal surfaces. Furthermore, at the $\bar{\Gamma}$ state, the interband contribution (bulk and from $\bar{M}_{1}$ state) to the energy-scaled linewidth $\left(\gamma /\left(E_{F}-E\right)^{2}\right)$ are energy independent (Fig. 2). This can be rationalized in terms of the phase space available for the transitions and the overlap between the initial (surface) and final (3D bulk or surface) wavefunctions, as occurs in a 3D degenerate electron gas model (DEGM) of an isotropic Fermi liquid. The sum of these two contributions is below $20 \%$ of the total $\gamma$. The case of the $\bar{M}_{2}$ state is more complicated since it is not a well-defined surface state as the other two states - it is located mainly at the third and fourth atomic layers ${ }^{13}$ - and it has one more decay channel, that is, electron transitions from the $\bar{M}_{1}$ state. For this state the intraband contribution is only $10 \%$ of the full decay rate, but interband transitions from the $\bar{M}_{1}$ state accounts for $38 \%$ comparable to the $44 \%$ contribution from bulk states. These results indicate that even for a very weak surface state inter- and intraband surface transitions provide more than $50 \%$ of the full linewidth. The reduction of the intraband contribution is responsible for the smaller linewidth at $\bar{M}_{2}$ as compared to the $\bar{\Gamma}$ state and thus the evaluated linewidth is closer to the expectations from a 3DDEGM (see Table I). Thus deviations from quadratic law stems from the peculiar character of the dynamicallyscreened surface-state transitions contributing to $\gamma$. This explains the different momentum and energy dependence in $\mathrm{Be}$ as compared to other metal surfaces $(\mathrm{Cu}, \mathrm{Ag}$, and $\mathrm{Mo}){ }^{8,11} \mathrm{It}$ can also be the explanation for the measured ${ }^{19}$ quasilinear dependence of $\gamma$ in graphite. ${ }^{20}$

As stated before, it is necessary to address the issue of how the low dimensionality of the surface influences the energy dependence of the hole linewidth and compare with the results from a $2 \mathrm{D}(3 \mathrm{D})$-DEGM. In the $3 \mathrm{D}-\mathrm{DEGM}$ the linewidth scales as $\gamma \propto\left(E_{F}-E\right)^{2},{ }^{5}$ while in 2D the dependence is modified to $\gamma \propto\left(E_{F}-E\right)^{2} \ln \left|E_{F}-E\right|^{6}$. As for Be, the 3D-DEGM gives $\gamma=90 \mathrm{meV}$ at $\bar{\Gamma}$ that is significantly smaller than our intraband contribution of $225 \mathrm{meV}$ but larger than the interband one $(40 \mathrm{meV})$. We attribute the first result to the absence of surface states in the 3D-DEGM (assumes only 3D bulk transitions and neglects band structure effects) whereas the latter is a direct consequence of the absence of a surface energy gap in 3D-DEGM. On the other hand, the 2D-DEGM gives $\gamma=610 \mathrm{meV}$ that is now substantially larger than our first principle value. This difference is a direct measurement of the different type of screening in the two models. The absence of the underlying bulk states in the 2D-DEGM strongly limits the screening of the electronelectron interactions. Therefore, larger linewidths are expected in this low-dimensional screening scenario for the hole decay. In fact, the computed energy-scaled linewidths in Fig. 2 exhibit a clear deviation from the expected constant value of a $3 \mathrm{D}$ system and the deviation increases as we approach the $E_{F}$. It is quite difficult to conclude the precise energy dependence of $\gamma$ (logarithmic or power law), but the computed data shows a strong deviation from standard quadratic law with an approximate energy dependence $\gamma \propto\left(E_{F}\right.$ $-E)^{\alpha}$ with $\alpha$ positive but smaller than two (the fitted power law dependence at $\bar{\Gamma}$ is about 1.6). The highly nonlocal electron self-energy (peaked at the surface) enhances the contributions from intraband transitions within the surface-state band ( $2 \mathrm{D}$ character). This effect is common to all metal surfaces, however the energy and momentum lifetime disper- 
sion is determined by specific surface-band structure and screening (provided mainly by the underlying bulk states).

We complement this theoretical study of the surface-state hole dynamics with detailed temperature-dependent angle resolved PES experiment on $\mathrm{Be}(0001)$ single crystal. ${ }^{21}$ The data were taken at a photon energy of $15 \mathrm{eV}$ and the light was incident at an angle of $30^{\circ}$ from the sample normal in the vertical plane. The combined energy and angular resolution of the photons and the analyzer was set to $170 \mathrm{meV}$ and $\pm 0 \times 10 \mathrm{mR}$ (corresponds to $\delta k_{\|}=0.013 \AA^{-1}$ ), respectively. More technical details can be found in Ref. 15.

The measured room temperature linewidth of the surface state at $\bar{\Gamma}$ was $390 \mathrm{meV}$ in good agreement with other room temperature PES results $440 \mathrm{meV}$ (Ref. 12) and $400 \mathrm{meV} .{ }^{15}$ It was found that the linewidth decreases as the surface state disperses towards $E_{F}$. This suggests that the impurity/defect contribution to the spectra is small at the $\bar{\Gamma}$ point. ${ }^{22,23}$ In Fig. 3 we show the summary of all the PES data. First, we fitted the spectra to a Lorentzian plus a linear background. To extract the electron-phonon contribution, the resulting linewidth versus temperature was fitted following the procedure in Refs. 15 and 24 using a simple 3D Debye model. The obtained electron-electron plus electron-impurity contribution to $\gamma$ at $\bar{\Gamma}$ is $281 \mathrm{meV} \pm 7.2 \mathrm{meV}$. The difference of 16 $\mathrm{meV}$ with respect to the computed linewidth can be tentatively attributed to impurity contribution. The value of 16 $\mathrm{meV}$ is similar to that found for the $s p$-surface state on other close-packed surfaces: $10-15 \mathrm{meV}$ for $\mathrm{Ag}(111)$ (Refs. 9 and 10) and $10-20 \mathrm{meV}$ for $\mathrm{Cu}(111) .^{25}$

The main results (not presented here) of the temperature dependent study of the $\vec{\Gamma}$ surface state at different binding energies (BE) along $\overline{\Gamma K}$ are as follows. The absolute width decreases with decreasing $\mathrm{BE}$ (the room temperature width at $\mathrm{BE}=0.35 \mathrm{eV}$ is about $30 \%$ smaller than at $\mathrm{BE}=2.8 \mathrm{eV}$ ). As expected, the phonon contribution changes with $\mathrm{BE}$, at $\mathrm{BE}=0.35 \mathrm{eV}$ it is about $35-40 \%$ higher than at $\bar{\Gamma}$. As the absolute photoemission linewidth can be trusted only in the region where $d E / d k_{\|}=0$ (Ref. 26) we make a quantitative comparison with the theory only for the $\bar{\Gamma}$ point.

In conclusion, our first-principle calculations reconcile the existing discrepancy between the DEGM theory and experiment concerning the hole lifetime in the surface-state band of metal surfaces and provides a basic understanding of the energy and momentum dependence of the hole linewidth. The

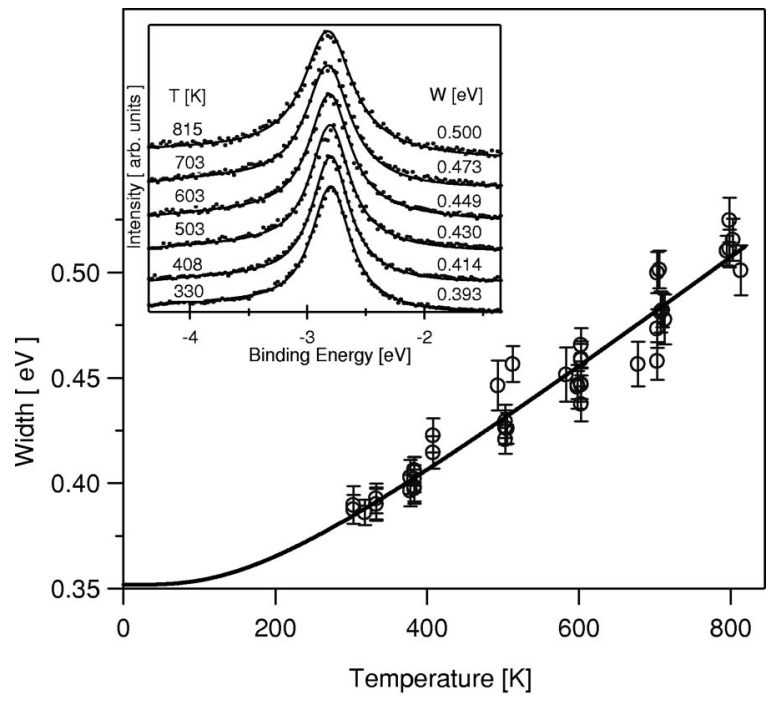

FIG. 3. Measured surface-state linewidth versus temperature at $\bar{\Gamma}$ (open circles). The line is the Debye model fit (see text for details). The inset shows the surface-state spectra at various temperatures (filled circles) with the fit using to a Lorentzian plus a linear background (line).

calculations show that the surface-band structure details are crucial in describing the decay mechanism of a hole in a surface state, in particular, the 2D surface-intraband transitions dominate the decay rate of well-defined surface states (enhanced by the spatial nonlocality of the self-energy), whereas screening is mainly controlled by the underlying three-dimensional electron system. Although this scenario has been obtained here for a particular surface, $\mathrm{Be}(0001)$, its physical origin is completely generally and needs to be always taken into account when dealing with quasiparticle dynamics in simple, noble, and transition metal surfaces.

We acknowledge support by the Basque Country University, Basque Hezkuntza Saila, Spanish MEC, HPRN-CT2000-00167 (NANOPHASE), and the Max Planck Research Award funds. We thank the $\mathrm{C}^{4}$ center for providing computing time. (T.B) is grateful to E. Jensen, S. L. Hulbert, and X. $\mathrm{L}$. Wu for useful discussions and the assistance during the experiments. The NSLS was supported by the Division of Material Sciences, U.S. DOE (Contract No. DEAC0276CH00016).
${ }^{1}$ E. W. Plummer and W. Eberhardt, Adv. Chem. Phys. 49, 533

(1982); F. J. Himpsel, Adv. Phys. 39, 1 (1983).

${ }^{2}$ N. Memmel, Surf. Sci. Rep. 32, 91 (1998).

${ }^{3}$ F. J. Himpsel et al., Adv. Phys. 47, 511 (1998).

${ }^{4}$ N. V. Smith, Phys. Today 54, 29 (2001).

${ }^{5}$ J. J. Quinn, Phys. Rev. 126, 1453 (1962).

${ }^{6}$ G. F. Giuliani and J. J. Quinn, Phys. Rev. B 26, 4421 (1982).

${ }^{7}$ R. Matzdorf, Surf. Sci. Rep. 30, 153 (1998); G. Nicolay et al., Phys. Rev. B 62, 1631 (2000).

${ }^{8}$ T. Valla et al., Phys. Rev. Lett. 83, 2085 (1999).

${ }^{9}$ J. Li et al., Phys. Rev. Lett. 81, 4464 (1998).
${ }^{10}$ J. Kliewer et al., Science 288, 1399 (2000); E. V. Chulkov et al., Surf. Sci. 454-456, 458 (2000).

${ }^{11}$ L. Bürgi et al., Phys. Rev. Lett. 82, 4516 (1999).

${ }^{12}$ R. A. Bartynski et al., Phys. Rev. B 32, 1921 (1985); U. O. Karlsson et al., Solid State Commun. 49, 711 (1984).

${ }^{13}$ E. V. Chulkov et al., Surf. Sci. 188, 287 (1987); P. J. Feibelman, Phys. Rev. B 46, 2532 (1992).

${ }^{14}$ E. W. Plummer and J. B. Hannon, Prog. Surf. Sci. 46, 149 (1994); L. I. Johansson et al., Phys. Rev. Lett. 71, 2453 (1993); P. T. Sprunger et al., Science 275, 1764 (1997).

${ }^{15}$ T. Balasubramanian et al., Phys. Rev. B 57, R6866 (1998); M. 
Hengsberger et al., Phys. Rev. Lett. 83, 592 (1999); S. LaShell et al., Phys. Rev. B 61, 2371 (2000).

${ }^{16}$ The Be pseudopotential is generated according to N. Troullier and J. L. Martins, Phys. Rev. B 43, 1993 (1991). Convergent results were obtained with a 20 Ry plane-wave cut-off for the wave functions and a $18 \times 18$ special $\mathbf{k}_{\|}$sampling points. In the evaluation of the dynamical screening we used $\sim 200$ bands (up to 50 $\mathrm{eV}$ ) above $E_{F}$ including reciprocal lattice vectors $\mathbf{g}$ in a sphere with an energy cutoff of 10 Ry.

${ }^{17}$ L. Hedin and S. Lundqvist, Solid State Phys. 23, 1 (1969); P. M. Echenique et al., Chem. Phys. 251, 1 (2000).

${ }^{18}$ I. Campillo et al., Phys. Rev. Lett. 83, 2230 (1999); 85, 3241 (2000); Phys. Rev. B 61, 13484 (2000); W.-D. Schöne et al., Phys. Rev. B 60, 8616 (1999).

${ }^{19}$ S. Xu et al., Phys. Rev. Lett. 76, 483 (1996); K. Ertel et al., Appl. Phys. B: Lasers Opt. 68, 439 (1999).

${ }^{20}$ C. Spataru et al., cond-mat/0107043; J. González et al., Phys. Rev. Lett. 77, 3589 (1996).

${ }^{21}$ Experiments were performed on the TGM beamline $\mathrm{u} 12$ at the National Synchrotron Light Source (NSLS), Brookhaven National Laboratory.

${ }^{22}$ S. D. Kevan, Phys. Rev. Lett. 50, 526 (1983); J. Tersoff and S. D.
Kevan, Phys. Rev. B 28, 4267 (1983).

${ }^{23}$ In the dilute impurity limit the temperature dependence of the impurity contribution is negligible as well as the electronelectron contribution, thus all the temperature dependence can be attributed to the electron-phonon contribution [B. A. McDougall et al., Phys. Rev. B 51, 13891 (1995)]. The main assumption in this procedure is that the electron-phonon contribution to the lifetime at the hole state binding energy of $2.78 \mathrm{eV}$ can be treated similarly to that of electrons close to the Fermi level.

${ }^{24} \mathrm{G}$. Grimvall, The Electron-Phonon Interaction in Metals (NorthHolland, New York, 1981).

${ }^{25}$ F. Theilmann et al., Phys. Rev. B 56, 3632 (1997).

${ }^{26}$ In photoemission, the electron-impurity contribution shows up in two ways: the first is the inherent contribution to the lifetime and the second is an artifact introduced by the $E(k)$ dispersion. ${ }^{22}$ The inherent contribution is independent of the binding energy, whereas the artifact contribution increases with decreasing binding energy for states dispersing like the $\bar{\Gamma}$ surface state. To extract the electron-electron contribution for a state with a different binding energy, one would then have to resort to some model for accounting for the impurity contribution at all binding energies. 